\title{
Measuring pedestrian trajectories using a pyroelectric differential infrared detector
}

\author{
Armitage, A., Binnie*, T. D., Kerridge, J., \& Lei, L. \\ School of Computing, Napier University \\ 10 Colinton Road, Edinburgh, EH10 5DT \\ *School of Engineering, Napier University \\ 10 Colinton Road, Edinburgh, EH10 5DT \\ Corresponding author: a.armitage@napier.ac.uk
}

\begin{abstract}
Low-cost, low-resolution infrared detectors have been used for measuring the trajectories of pedestrians. The detectors have been designed for counting the number of pedestrians crossing a line. The use of these detectors has been extended to provide complete trajectories across measurement areas approximately 3 Metres Square. This provides an effective way of rapidly measuring large numbers of pedestrian movements. Current work involves extending the effective area by combining trajectories from multiple detectors. Matching across detectors has been made difficult by the presence of edge effects. Progress is being made on the algorithms needed to track across fields of view.
\end{abstract}

\section{INTRODUCTION}

Accurate measurement of pedestrian trajectories is useful in a variety of situations. In retail environments, information about customer behaviour and preferences can be derived from knowledge of customer's movements. In crowded locations (such as sports venues, railway station concourses and so on) pedestrian movement influences safety concerns. Our primary concern is in the general planning of pedestrian movements within the urban environment (shopping malls, shopping streets, pedestrian concourses and the like). A related project at Napier University (Pedflow ${ }^{1}$ ) has been concerned with predicting pedestrian flows in such settings. Validation and verification of such models requires data of real pedestrian movements, both in the initial formulation of behavioural rules, and in the testing of the resultant models. Traditional methods of gathering such data are laborious, and difficult to automate. The overall aim of this project is to automate this process, allowing for the gathering of large amounts of data for use in model derivation and validation.

\section{DETECTING PEDESTRIANS}

Pedestrian movement can be monitored by a variety of means. Observers with clipboards can be used, though it is difficult to get detailed trajectory information in real time. If the flow can be restricted, break-beam technology can be used to count pedestrians passing by. This method becomes very inaccurate as the width of area covered increases. This is because several people may pass through side-by-side, with inevitable masking of the beam.

Video recording is a very useful technique, as it allows scenes to be replayed, or slowed down, while an observer notes the trajectory of individuals. Unfortunately, there is currently no reliable method for automatically extracting behaviour using software. Urban scenes at visible wavelengths are often very confused and cluttered. Changes in lighting and shadows (as the sun goes behind a 
cloud, for instance) all go to reducing the accuracy of software methods. Software is available that can help with the extraction of detailed information, but it must be used by an operator who can guide the process. Although time-consuming, this can be a reliable method for extracting detailed measurements ${ }^{2}$.

\section{THE INFRARED DETECTOR}

Long-wavelength infrared detectors are difficult to fabricate in arrays. Semiconductor arrays of Mercury Cadmium Telluride, Indium Antimonide and the like have been used for some time in astronomical and other applications, but at a high cost. This is partly due to the costs of the exotic semiconductor, but also to the cooling system that is needed for noise reduction. Recently, uncooled arrays of microbolometers have become available, leading to lower system costs. Despite this, the cost of an infrared detector is still of the order of 100 times that of a detector working at visible wavelengths.

The detectors used in this study have been developed by Irisys ${ }^{3}$ as an attempt to provide a relatively low cost infrared array detection device (the detectors described on the company web site are actually a later model than the ones used here). The detector is based on an array of pyroelectric ceramic detection elements ${ }^{4,5}$. The array is a square format, with 16 rows and 16 columns of pixels. This is a relatively small number of elements, resulting in a low-resolution image (figure 1). However, the cost of the detector is of the order of $1 / 20^{\text {th }}$ of that of a traditional IR array.

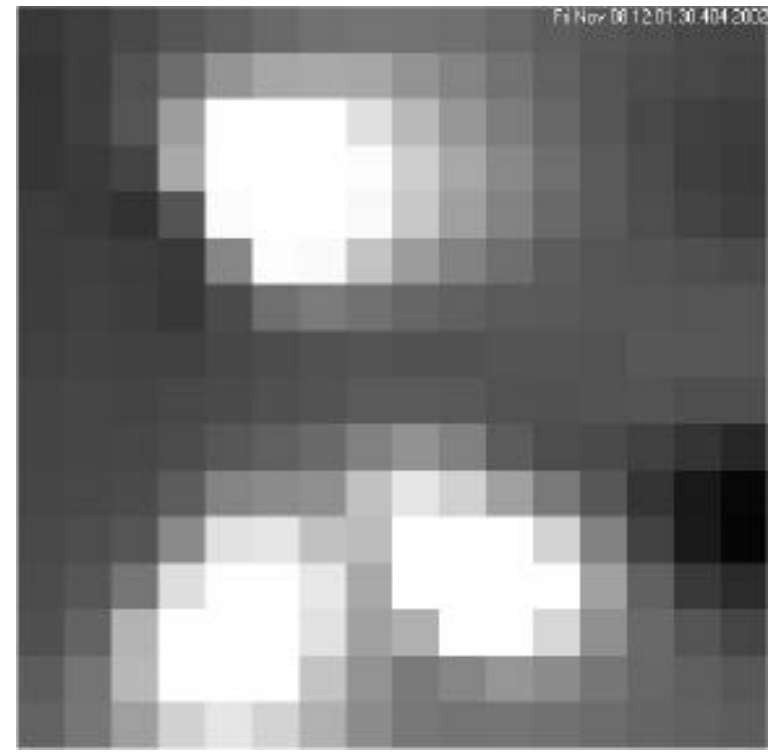

(a)

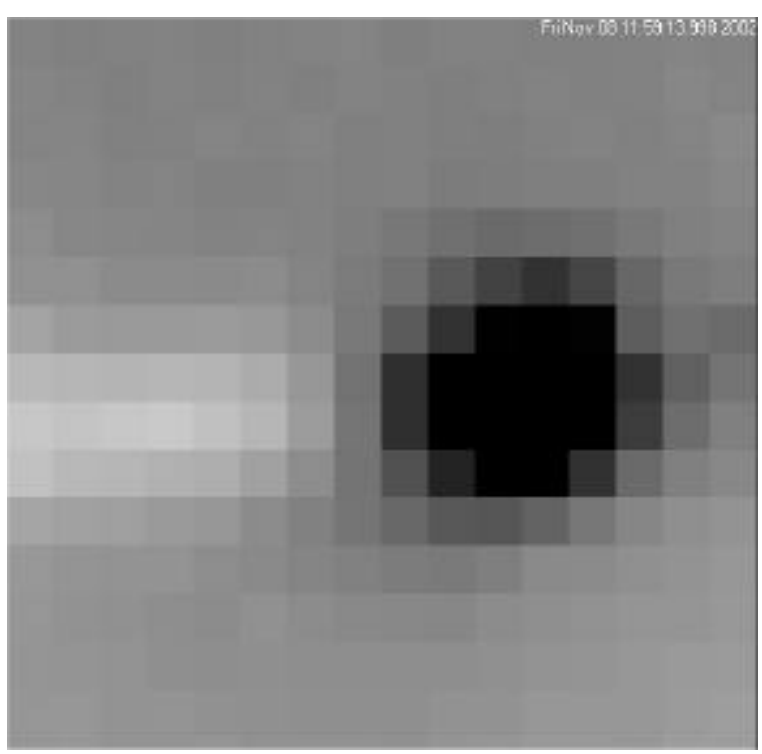

(b)

Figure 1. In image (a), three pedestrians are moving from right to left. This is the normal situation, where pedestrians are hotter than the background. In (b), a pedestrian has just entered an indoors environment and is moving left to right. Taken in Edinburgh in November, the outer layers of the pedestrian are colder than ambient, and they show as a dark mass. Note the faint warm 'wake'. This is caused by the detector being sensitive to changes in temperature. In this case the change from cold pedestrian to warm ambient produces a temperature increase, i.e. an apparent warm wake.

Unusually for a pyroelectric detector, there is no optical chopper. Pyroelectric devices respond to change in temperature. When imaging an infrared scene, pyroelectric detectors view the radiation after it has been chopped, usually by a rotating mechanical device. The detector then effectively measures the difference between the scene temperature and the temperature of the chopper blades. To keep the system costs down, the Irisys detector contains no chopper (a more expensive version is available that includes a mechanical chopper). This means that the detector responds only to changes (positive or negative) in the scene temperature. For pedestrians walking at normal rates, 
this is not a problem. The time constant of the detector is several seconds, so a pedestrian that stands motionless disappears from the scene after a few seconds, and this does cause difficulties when monitoring queues.

In normal use, the detector is suspended at a height of about 3 metres above the area to be measured. At ground level, a square approximately 3 metres by 3 metres is imaged. The images can be relayed via a standard serial connection to a computer for display and recording. The detector is intelligent, with an on-board DSP chip that processes the image. Pedestrians are monitored by fitting an ellipse to candidate 'blobs' in the image area. The DSP then sends, via the serial interface, details of candidate targets. These details include position, change in position, status information, and the characteristics of the ellipse that has been fitted to the target. The status information shows whether the target is hotter or colder than ambient, whether it is a new target in the scene, and whether the candidate target has fragmented (this happens, for instance, if pedestrians hold an arm out). Figure 2 shows a typical scene with a number of pedestrians that have been identified.

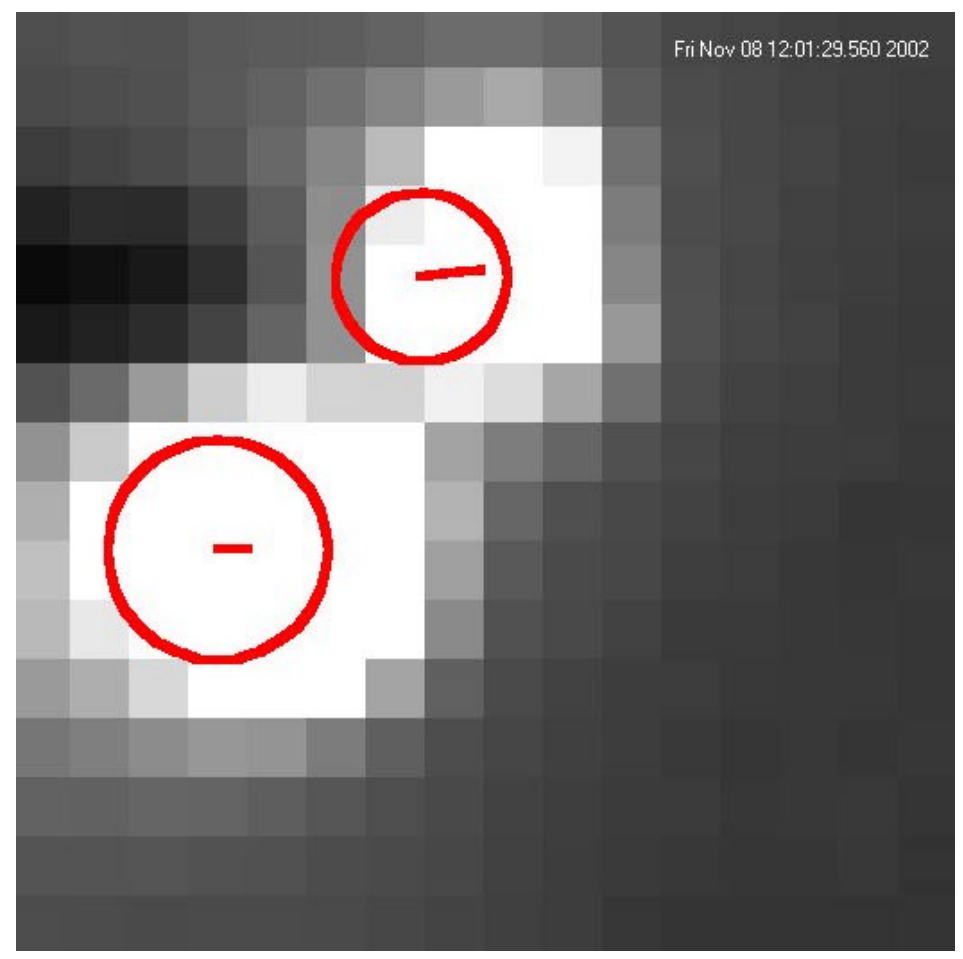

Figure 2: Two pedestrians moving to the right. Note the visual indication by a short bar of the current velocity. There is a slight delay between the time at which the image is taken, and the time at which the ellipses are measured. This results in an apparent mis-registration between the two on the image. Although this occurs on the displayed information, the recorded trajectory information is actually accurate.

\section{Matching between detectors}

The pedestrian counter is normally intended for use counting targets moving across a straight line (horizontal or vertical in the field of view). As such, it

functions well counting people entering supermarkets, moving through corridors, and passing across railway and airport concourses. In our application area, we are interested in pedestrian movements within an urban environment (e.g. shopping streets). We would like to be able to derive trajectories for pedestrians as they move in arbitrary directions through a scene. In principle, tracking a pedestrian in the field of view of a single camera is not difficult. The real difficulty arises when the scene is so large that it has to be covered by multiple cameras. Tracking between adjacent cameras is not easy, as the low resolution of the image means that it is not possible to identify individuals. Matching has to be done on the basis of trajectories, and matching across boundaries. It would be possible to overlap fields of view, so that pedestrians appear simultaneously in the fields of view of two detectors. However we wished to avoid this, as it substantially reduces the area covered by the detectors.

\section{Limitations of the detector}

The obvious limitation of the detector is the low resolution. As far as our application is concerned, this is not a great problem. The use of an algorithm to fit ellipses round targets means that the average centroid of the target is located with sub-pixel accuracy. With a field of view of 3 metres, 
each pixel spans approximately $19 \mathrm{cms}$, and the location of the target is known to an accuracy of the order of $10 \mathrm{cms}$.

The detector has a wide field of view in order to cover a reasonable area on the ground, without having to be mounted too high up. This wide angle inevitably results in distortion, as can be seen in figure 3. This distortion is not a problem, as it can be allowed for.

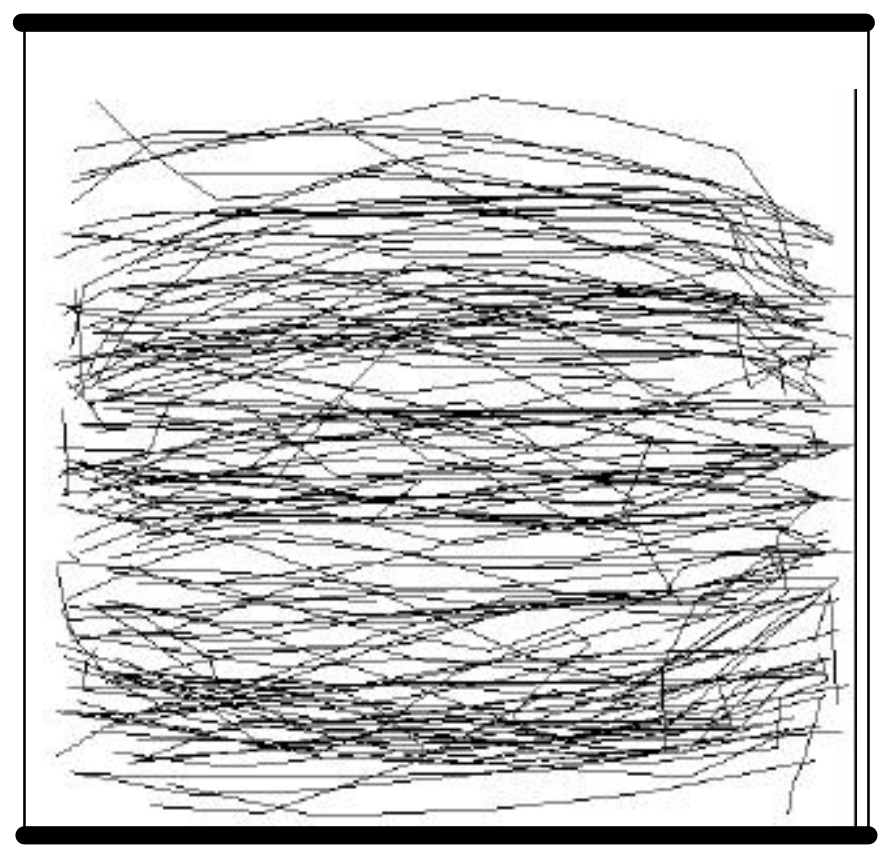

Figure 3. The effect of the wide-angle lens can be seen in this cumulative plot of trajectories. Although this has been taken in a straight corridor, with the bulk of pedestrians moving from side to side, the apparent width of the corridor is less at the left and right hand sides than in the centre.

Another problem is the low frame rate of the detector. Internally, the DSP runs at a frame rate of $33 \mathrm{~Hz}$. Limitations in the communications mean that only every tenth frame can be transmitted to the PC where the images are displayed, recorded and processed. For pedestrians walking briskly through the field of view, this means that typically only four or five points are recorded as they pass through. This problem is compounded by the fact that the DSP can not always be confident of identifying a target as it enters a frame (the pedestrian may be half in and half out of the frame). Consequently, some of the frames at the edges are marked as invalid. This effect can be seen in figure 4. Irisys have recently introduced a new version of the detector, the IRC 1004. This has an improved communications link, and should not suffer from this limitation.

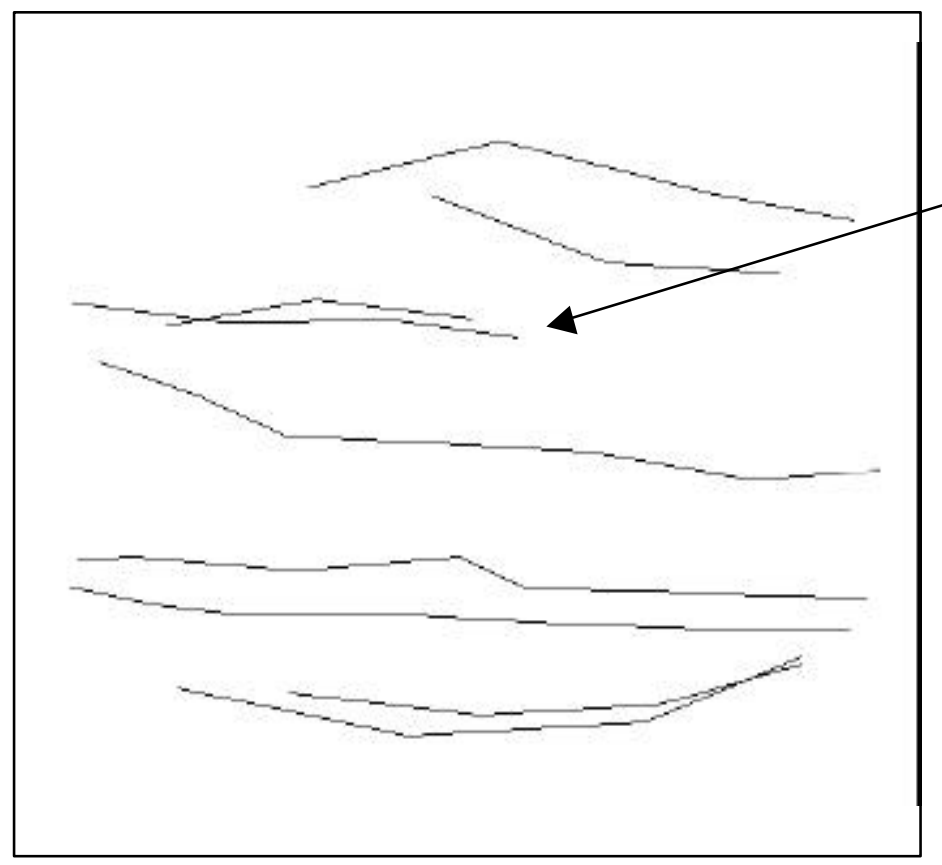

Figure 4. Trajectories are constructed from a relatively small number of points. Some trajectories start a long way in from the edge. This is normally due to the DSP in the detector rejecting the first or last point.

The area covered by a single detector is approximately 3 metres square. Ideally, we would like to be able to cover larger areas by tracking pedestrians between detectors. To do this, we have been working on matching targets across the boundaries. There is not enough detail in the images to be able to recognise individuals, so decisions must be based purely on the matching of trajectories between fields of view. The edge effects mentioned above have made this harder than we had originally anticipated, as we have to search quite far in for a candidate target. Initially, we follow a target out of one field of view, then look for candidate targets appearing within three to four pixels 
of the boundary of the next field of view. Targets that appeared within the correct area within one second were considered for matches. However, this missed matches because first and last readings were nor valid, resulting in a larger than expected gap between fields of view. Figure 5 illustrates the improvement that results when increasing the permissible time gap between a target disappearing from one field of view, and appearing in a new field. The number of matches increases up to about 1.5 seconds, and then does not increases further, as all possible candidate targets have been found within this time. Although adjusting the matching algorithm has improved the number of matches found, we still lose track of too many targets between frames (success rates are of the order of $60 \%$ ). Current work is therefore focussing on improving the algorithms further. Our initial plan was to be able track across four detectors. As this means some pedestrians will cross three boundaries, we will need to improve the success rate for the technique to be successful. However, the most recent versions of the detector produce more trajectory points per second, and this should improve the readings at the edge of the fields of view.

Figure 5: Effect of increasing the permissible time gap between a target moving from one field of view to the next.

\section{Results}

The detector works well at gathering large numbers of pedestrian trajectories. Figure 6 illustrates measurements taken over a 10 minute period in a crowded corridor. An obstruction detector in the time. On occasions, seven or eight pedestrians may be in the field of view at the same time; the detector manages to track them all successfully. Although coordinate data is

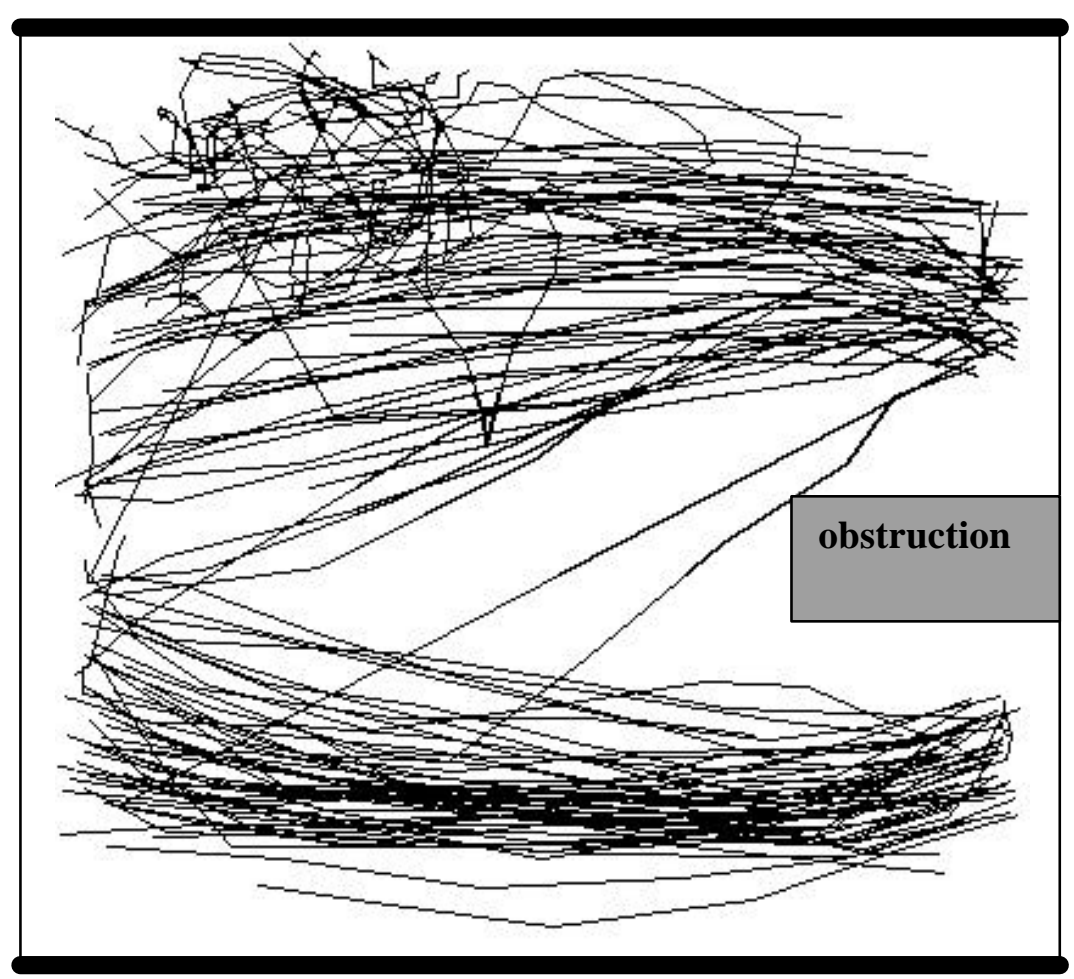
available for the individual trajectories, a simple plot such as that of Figure 6 provides a good impression of the overall flow. Ultimately we want to be able to derive accurate measurements that can be used as the basis of pedestrian behaviour in the agentbased models we are currently developing ${ }^{1}$.

Figure 6. Flow of pedestrian around an obstruction in a corridor. Note the appearance of a 'loitering' pedestrian in the upper left quadrant. 


\section{Conclusion}

The Irisys detector efficiently measures pedestrian movements over an area of approximately 3 to 3.5 metres square. The data that is produced is in a readily analysed format. Slight distortions in the coordinates due to the optical arrangement are not important. The main limitation is the errors found in some measurements at the edges of the field of view. This leads to difficulties when trying to correlate the movements of pedestrians across multiple fields of view. New versions of the detector should not have these problems

\section{Acknowledgements}

Funding for this project has been provided under the LINK Future Integrated Transport Project: FIT002. The cooperation of Irisys in providing equipment and information is also gratefully acknowledged.

\section{References}

1. Kukla, R. \& Kerridge, J., "PEDFLOW: Development of an Autonomous Agent Model of Pedestrian Flow", 80th Annual Meeting TRB, Washington, DC, USA, 2001,

2. Kukla, R. \& Kerridge, J., "Laying the foundations: the use of video footage to explore pedestrian dynamics in PEDFLOW", Pedestrian Evacuation and Dynamics, 2001 Duisburg, Germany, 2001 ,

\section{3. www.irisys.co.uk}

4. Hollock, S,. Galloway, J. L., “ Smart Sensors Using Array Based Infrared Detectors”, Sensors and their applications XI/ISMCR 2001 - Institute of Physics, City University London, September 2001

5. Mansi, M. V., Porter, S. G. Galloway, J. L. \&. Sumpter N., "Very low cost infrared array based detection and imaging systems" (SPIE) Aerosense 2001, Orlando, Florida USA, 17-19 April 2001 